\title{
TRATAMIENTO COGNITIVO-CONDUCTUAL GRUPAL DEL TRASTORNO DE PÁNICO CON O SIN AGORAFOBIA EN UN CENTRO DE SALUD MENTAL PÚBLICO
}

\author{
AsCensión GARrigA ${ }^{1}$, JUlio C. MARTÍN ${ }^{1}$ Y CONCHA LÓPEZ SOLER ${ }^{3}$ \\ ${ }^{1}$ Servicio Murciano de Salud, Hospital Psiquiátrico, Murcia \\ ${ }^{2}$ Facultad de Psicología, Universidad de Murcia y Hospital V. Arrixaca, Murcia
}

\begin{abstract}
Resumen: La eficacia de la terapia cognitivo-conductual (TCC) en el tratamiento del trastorno de pánico (TP) con o sin agorafobia está bien documentada; sin embargo, se conoce menos su efectividad en formato grupal. El propósito de este estudio es investigar la efectividad de una combinación de las principales técnicas utilizadas en una versión específica de TCC en formato grupal para este trastorno: el Modelo de Control del Pánico. El tratamiento consistió en 12 sesiones grupales semanales siguiendo la guía propuesta por Martín (2007) para aplicar en grupo el programa de Barlow (1988). Todos los pacientes tuvieron ganancias significativas en todas las dimensiones evaluadas con alto grado de remisión de sintomatología fisiológica de las crisis de pánico y mejora significativa en los síntomas asociados. Los resultados sugieren la recomendación de este modelo de tratamiento para el abordaje de los trastornos de pánico en una consulta pública de salud mental.
\end{abstract}

Palabras clave: Trastorno de pánico, agorafobia, terapia cognitivo conductual grupal, efectividad.

\section{Group cognitive-behavioral therapy for panic disorder with or without agoraphobia in a public mental health center}

\begin{abstract}
The efficacy of cognitive-behavior therapy (CBT) in the treatment of panic disorder (TP) with or without agoraphobia is well documented, but less is known about group CBT effectiveness. The purpose of this study is to investigate the effectiveness of a combination of the main techniques used in a specific version of CBT for the treatment of this disorder: the Control Panic Program. The treatment consisted of 12 weekly group sessions, following the guidelines proposed by Martin (2007) to implement the Barlow program in a group format (Barlow 1988). All patients showed significant gains in all dimensions assessed, with a high degree of remission in panic attacks' physiological symptoms and significant improvement in associated symptoms. Results warrant recommending this model for the treatment of panic disorder in public mental health consultancies.
\end{abstract}

Keywords: Panic disorder, agoraphobia, group cognitive-behavior therapy, effectiveness.

\section{INTRODUCCIÓN}

Los ataques de pánico son periodos concretos de intenso miedo y malestar, acompañados de, al menos, cuatro síntomas de ansiedad físicos o psicológicos. Alrededor de $2 / 3$ de los pacientes con trastorno de pánico $(\mathrm{TP})$ desarro-

Recibido: 18-marzo-2008; aceptado: 29-enero-2009.

Correspondencia: Julio C. Martín, Subdirección de Salud Mental, Servicio Murciano de Salud, Hospital Psiquiátrico, Crta. Mazarrón s/n. 30120 el Palmar (Murcia).

Correo-e: julioc.martin@carm.es llan agorafobia, definida por miedo a sitios o situaciones de las que resulta difícil escapar o recibir ayuda, en caso de sufrir un ataque de pánico. Situaciones típicas son alejarse de casa, estar en un cine o utilizar un trasporte público. Según estudios comunitarios a gran escala alrededor de un $10 \%$ de la población adulta experimenta algún ataque de pánico, aunque en la mayoría de casos no llega a desarrollarse un TP (Robins et al., 1984).

El TP con o sin agorafobia supone un importante problema de salud pública (Margraf, 
Barlow, Clark y Telch, 1993). Su prevalencia a lo largo de la vida es aproximadamente del 3\% (Barlow, Gorman, Shear y Woods, 2000). Datos epidemiológicos obtenidos en 40.000 sujetos de diez países encuentran unas tasas de prevalencia del 1,4\% al 2,9\% (Weissman, Bland, Canino, Faravelli y otros, 1997) y, según el DSM-IV (Diagnostic and Statistical Manual of Mental Disorders), la prevalencia anual se sitúa entre el $1,5 \%$ y el $3,5 \%$.

La edad de inicio más frecuente del trastorno es alrededor de los 20 años y existe un riesgo dos veces mayor de padecerlo por parte de las mujeres (Weissman et al, 1997). El estrés psicosocial previo no se asocia de forma consistente a la ocurrencia del TP, sólo la dimensión social del estrés predeciría la existencia o no de agorafobia (Sandín, Rodero, Santed y García-Campayo, 2006). Aunque su evolución tiende a fluctuar, frecuentemente es crónico y, en su curso natural, muestra un bajo porcentaje de remisiones (Keller et al., 1994; Yonkers et al., 1998) provocando una importante reducción en la calidad de vida. Afecta al ajuste matrimonial y a la independencia económica disminuyendo la sensación de auto-eficacia, con las importantes consecuencias negativas que esto supone (Weissman, 1991). Todo ello, en opinión de Margraf et al. (1993), explica el hecho de que estos pacientes busquen ayuda profesional con mayor frecuencia que con cualquier otro trastorno. El pronóstico a largo plazo, sin un tratamiento adecuado, es peor que el de la depresión mayor (Botella, 2001).

Las personas con TP frecuentan los servicios de urgencias y de medicina general presentado un porcentaje muy alto de síntomas cardiacos inexplicables, vértigos y molestias intestinales (Barlow et al, 2000). También resulta más probable su hospitalización por problemas físicos y utilizan más psicofármacos (Klerman, Weissman, Oullette, Jonson y Greenwald, 1991; Markowitz, Weissman, Oullette, Lish y Klerman, 1989). Los costes económicos y sociales del TP son considerables. Sin embargo, el TP tratado adecuadamente puede producir una mejoría clínica de hasta el 94\% (Barlow et al., 2000).

El TP presenta alta comorbilidad con otras alteraciones psicopatológicas. Respecto al Eje
I del DSM-IV (APA, 1994) entre un 65\% y un $88 \%$ padecen otros trastornos, siendo los más frecuentes los de ansiedad y del estado de ánimo, patología que se incrementa a medida que se agrava la evitación agorafóbica (Barlow, DiNardo, Vermilyea, Blanchard, 1986; Sanderson, DiNardo, Rappe y Barlow, 1990, Brown y Barlow, 1992; Starcevic, Uhlenhuth, Kellner y Pathak, 1992). También aumenta el abuso de sustancias y el riesgo de suicidio (Brown y Barlow, 1992; Weissman, Klerman, Markowitz y Oullette, 1989). Respecto al Eje II del DSMIV, entre un $40 \%$ y un $50 \%$ de pacientes cumplen criterios para el diagnóstico de uno o más trastornos de personalidad (Mavissakalian, 1990; Pollack, Otto, Rosenbaum y Sachs, 1992).

La terapia cognitivo-conductual (TCC), es altamente eficaz en el tratamiento del trastorno de pánico con o sin agorafobia como demuestran varios meta-análisis (Clum y Surls, 1993; Michelson y Marchione, 1991: Wolfe y Márser, 1994) y confirman diferentes ensayos clínicos (Craske, Brown y Barlow, 1991; Margraf et al. 1993: Ost, Westling y Hellstrom, 1993; Telch et al., 1993), así como el National Institute of Mental Health (NIMH) agencia del Nacional Institue of Health de USA (1991). Se han desarrollado varias versiones de TCC para TP combinando estrategias con objetivos específicos: (a) Reestructuración cognitiva para corregir errores de interpretación de las sensaciones corporales como eventos peligrosos; (b) exposición en vivo a las situaciones o estímulos temidos para desconfirmar la experiencia aprendida y los automatismos mentales relacionados (Jacobson y Revenstorf, 1988) que ayudan a superar la evitación agorafóbica (Marks, 1987); y (c) animar al trabajo entre sesiones para generalizar y reforzar los resultados fuera del centro de salud mental (Galassi, Quercioli, Carismas, Niccolai y Barciulli, 2007). Estos tratamientos tienen elevadas tasas de éxito y se dispone de manuales de tratamiento para el terapeuta y de manuales de autoayuda para los pacientes (Beck y Zebb, 1994).

La American Psychological Association (APA), en el grupo de promoción y diseminación de procedimientos psicológicos (1995) y, posteriormente, en la guía clínica sobre inter- 
venciones (APA, Task Force on Psychological Interventions Guidelines), teniendo en cuenta las recomendaciones sobre exigencias metodológicas (Nathan y Gorman, 1998), clasifica los tratamientos psicológicos en dos ejes: por su eficacia y por su utilidad clínica. En el primer eje, los tratamientos bien establecidos para el TP son: (a) el tratamiento del control del pánico (TCP), desarrollado por el grupo de Barlow (Barlow y Cerny, 1988; Barlow y Craske, 1989,1994) que describimos con detalle en el apartado sobre procedimiento; y (b) la terapia cognitiva para el TP. El grupo de Clark (1986, 1989 ) incluye varios componentes para identificar y someter a prueba la adecuación de las interpretaciones catastrofistas y sustituirlas por otras más realistas. Incluye un componente educativo, otro cognitivo, «experimentos» para inducir sensaciones temidas con recomendaciones para abandonar «conductas de seguridad» y tareas para casa. La diferencia más notable entre ambos enfoques es que en el programa de Barlow se da un gran énfasis a la exposición a las sensaciones interoceptivas y en el programa de Clark al componente cognitivo.

Respecto al eje sobre utilidad clínica o validez externa (segundo eje) la efectividad de los actuales tratamientos para el TP está bien establecida. Los investigadores del NIHM de USA recomiendan desarrollar modos de aplicación que aumenten su disponibilidad (Lidren et al., 1994). Botella (2001, 2004), en su exhaustiva revisión, indica la importancia de replicar los estudios de efectividad y que estos tratamientos eficaces estén disponibles de forma que permitan una adecuada aplicación por parte de otros profesionales.

En lo que concierne a las terapias cognitivoconductuales grupales en el tratamiento de los trastornos de ansiedad, los estudios son escasos ya que la mayoría se han realizado de forma individual (Belfer, Muñoz, Schachter y Levendusky, 1995; Martisen, Olsen, Tonset, Nyland y Aarre, 1998; Penava, Otto, Maki y Pollack, 1998; Telch et al., 1993).

Telch et al. (1993) aplicaron el tratamiento del control del pánico (TCP) de Barlow en grupo, demostrando su eficacia. Los pacientes recibieron TCP durante 8 semanas en grupos de cuatro a seis pacientes, comparándolo con con- troles en lista de espera. Al concluir el tratamiento, el 85\% del grupo experimental estaba libre de crisis de pánico, frente al $30 \%$ del grupo control. Los tratados también mejoraban significativamente en índices de ansiedad, agorafobia, depresión y miedo al miedo. Los resultados se mantenían a los seis meses. (Telch et al., 1993)

En Indiana (Estados Unidos) se llevó a cabo un estudio de efectividad en un centro de salud mental y compararon sus resultados utilizando una estrategia de benchmarking con los resultados de eficacia de las clínicas de investigación (Wade, Treat y Stuart, 1998). El 87\% de los pacientes permanecían libres de crisis al finalizar el tratamiento y se producía un descenso significativo de la ansiedad anticipatoria y generalizada, de la evitación agorafóbica y de los síntomas de depresión. Estos resultados se mantenían al año de seguimiento (Stuart, Treat y Wade, 1998).

En nuestro entorno se han comparado los resultados de la misma intervención en un servicio universitario de psicología y en un centro de salud mental (García-Palacios et al., 2002); anteriormente este equipo había adaptado el modelo de Clark y Salkovskis para intervención grupal (Ballester y Botella, 1992). García-Palacios et al. (2002), obtienen resultados similares, pero con un índice de abandono muy bajo ( $0 \%$ comparado con el $26,4 \%$ de Wade et al.). Este resultado es comparable al de las clínicas universitarias (Barlow y Craske, 1989; Telch, et al., 1993). Más recientemente, Sharp, Power y Swanson (2004) comparan la eficacia y aceptabilidad de la TCC grupal e individual en atención primaria obteniendo unos índices de abandono muy altos en la intervención grupal (47\%). En un estudio llevado a cabo en Italia también se obtienen mejorías significativas en un tratamiento grupal en un centro de salud mental (14 sesiones de dos horas): al finalizar el tratamiento el $54,2 \%$ de los tratados estaban libres de crisis de pánico (Galassi et al., 2007). Las características de estos estudios, que servirán para comparar nuestros resultados mediante una estrategia de benchmarking, se resumen en Tabla 1.

Estos datos indican que la terapia grupal puede aplicarse de forma efectiva en la clínica 
Tabla 1. Características de los programas de tratamiento comparados

\begin{tabular}{|c|c|c|c|c|c|c|}
\hline & $\begin{array}{c}\text { Garriga, } \\
\text { Martín y } \\
\text { López-Soler } \\
\text { (este estudio) }\end{array}$ & $\begin{array}{c}\text { Wade et al. } \\
\text { (1998) }\end{array}$ & $\begin{array}{c}\text { Garcia- } \\
\text { Palacios et al. } \\
\text { (2002) }\end{array}$ & $\begin{array}{c}\text { Galassi et al. } \\
\text { (2007) }\end{array}$ & $\begin{array}{c}\text { Telch et al. } \\
\text { (1993) }\end{array}$ & $\begin{array}{c}\text { Clark et al. } \\
\text { (1994) }\end{array}$ \\
\hline Tipo de estudio & Efectividad & Efectividad & Efectividad* ${ }^{*}$ & Efectividad & Eficacia & Eficacia \\
\hline Tratamiento & TCP & TCP & TC/ TCP & TCC & TCP & $\mathrm{TC}$ \\
\hline $\begin{array}{c}\mathrm{N}^{\mathrm{o}} \text { sesiones / } \\
\text { duración }\end{array}$ & $12 / 90^{\prime}$ & $15 / ?$ & $14 / 90^{\prime}$ & $14 / 120^{\prime}$ & 12/ 90' & 12 \\
\hline $\begin{array}{l}\text { Pacientes por } \\
\text { grupo }\end{array}$ & 10-12/ grupo & $i ? /$ grupo & 5-6/ grupo & 10-12 / grupo & 4-6 / grupo & individual \\
\hline $\begin{array}{l}\text { Abandonos } / n \\
\text { (\% abandonos) }\end{array}$ & $\begin{array}{c}3 / 35 \\
(8,57 \%)\end{array}$ & $\begin{array}{c}29 / 110 \\
(26,37 \%)\end{array}$ & $\begin{array}{l}0 / 25 \\
(0 \%)\end{array}$ & $\begin{array}{c}15 / 76 \\
(22,3 \%)\end{array}$ & $\begin{array}{l}0 / 34 \\
(0 \%)\end{array}$ & $\begin{array}{c}1 / 20 \\
* *\end{array}$ \\
\hline
\end{tabular}

Nota . TC = Terapia cognitiva; TCP = Terapia de control del pánico de Barlow; TCC = Terapia cognitivo-conductual.

* Una parte de la muestra en clínica universitaria. **Se reemplazaron los abandonos para mantener los grupos comparables.

habitual, aunque, de partida, nuestros grupos son más numerosos (hasta 15 pacientes citados por grupo) lo que disminuiría su coste. Según los estudios sobre salud mental basada en la evidencia, el tratamiento grupal puede aplicarse en un servicio de salud pública, tiene bajo coste, requiere poco entrenamiento del equipo y permite optimizar recursos (Leveni, Mazzoleni y Piacentini, 1999). El Nacional Health Service Economic Evaluation Database (NHS EED), en un análisis de coste efectividad del de psicoterapia versus tratamiento combinado (farmacoterapia y psicoterapia) para el TP con agorafobia, resalta que el tratamiento combinado es más caro que la TCC sola, aunque las dos estrategias son igualmente eficaces (Marchand, Germain, Reinharz, Mainguy y Landry, 2004). A largo plazo la razón coste-efectividad se incrementa a favor de la TCC sola.

Nos proponemos replicar los estudios que han utilizado el TCP grupal de Barlow y Craske (1989) para el TP con o sin agorafobia en las condiciones clínicas habituales de un centro de salud mental público, con pacientes derivados por los propios profesionales del centro. Esperamos comprobar la adaptación del programa a cualquier persona que lo necesite, independientemente de los años de evolución del TP, de si sigue tratamiento farmacológico de más o menos duración, y/o de su nivel económico y cultural. En el Centro de salud Mental (CSM) de San Andrés de Murcia se aplica el TCP grupal de forma ininterrumpida desde 1989, por lo que podemos considerarlo un recurso asistencial consolidado. Aquí se inició la formación en este programa para otros profesionales y psicólogos internos residentes (PIR; Martin, 2007) y lo consideramos un centro de referencia. Para un servicio público con pocos psicólogos y gran demanda de asistencia, este tipo de alternativas terapéuticas es de gran interés.

Antes de la primera cita con psicología clínica todos los pacientes pasan por consultas médicas de primaria o especializada. Cuando son derivados al grupo gran parte siguen ya tratamiento farmacológico, lo que impone mayoritariamente el tratamiento combinado. El paso por atención primaria retrasa la oferta de TCC, ya que normalmente se instaura un tratamiento farmacológico. Probablemente, un grupo de pacientes mejora lo suficiente como para proponer un alta médica. Por tanto, lo esperable es que sólo los que padezcan un trastorno más grave o alguna sintomatología asociada, sean derivados a Salud Mental (Martín, 2007).

\section{MÉTODO}

\section{Participantes}

Los 39 participantes del estudio, siguiendo el procedimiento habitual, fueron remitidos por su médico de familia. Un psiquiatra o psicólogo 
clínico del CSM realizaron una entrevista clínica y establecieron un diagnóstico según criterios CIE-10 (OMS, 1992). Si los pacientes cumplían criterios diagnósticos de TP con o sin agorafobia eran derivados al grupo. En la primera sesión la terapeuta confirmaba si los pacientes manifestaban o no los síntomas que configuran el diagnóstico CIE-10. Para constituir los grupos no se siguió ningún criterio en cuanto a edad, nivel cultural, profesión, etc. No se dieron instrucciones específicas a los psiquiatras o médicos de familia sobre las pautas a seguir con la medicación, que podían cambiar según su criterio y evolución del paciente durante el tratamiento grupal. En el grupo se dio la única instrucción al paciente de que si deseaba disminuir la medicación, bien por mejoría, bien en la fase de provocar sensaciones, lo consultara siempre con su médico y siguiera las pautas de descenso que le indicara.

Se establecieron los 3 siguientes criterios de inclusión y exclusión: (1) Los pacientes eran seleccionados según el orden de derivación interna al grupo si cumplían los criterios diagnósticos CIE-10 de trastorno de pánico (F.41.0) o agorafobia (F.40). (2) Los pacientes eran excluidos por: trastornos de la personalidad como diagnóstico principal. En el caso de la esquizofrenia, trastornos bipolares y otros trastornos mentales graves que a veces presentan crisis de ansiedad, debe tratarse en primer lugar el trastorno principal. Sólo si está controlado y en fase estable, se valorará el tratamiento específico del pánico o la agorafobia si persisten. También se debe excluir el trastorno hipocondríaco y los trastornos obsesivos si son el principal diagnóstico. (3) Se excluye al paciente que expresa sus preferencias por el tratamiento farmacológico, o que no puede acudir a sesiones semanales. También cuando hay una ganancia secundaria muy importante por el trastorno (p.ej., baja por enfermedad y que la mejoría supone volver a su puesto, en el que sufría acoso laboral según el paciente).

Se establecieron los siguientes criterios de asistencia, no asistencia y abandono: (1) Asistencia: pacientes que acuden de forma regular al grupo y asisten al menos al $80 \%$ de las sesiones. (2) No asistencia: acuden a la primera sesión de grupo (informativa) y faltan después, habiéndolo comunicado o no. Y (3) abandono: acuden como mínimo a dos sesiones de grupo y menos del $80 \%$ restante (Martín, 2007).

Durante un periodo de 9 meses se seleccionó el grupo de tratamiento, el cual se constituyó mediante pacientes remitidos por los psiquiatras y psicólogos clínicos del CSM. De estos pacientes, decidieron no asistir un total de cuatro, dos por incompatibilidad con su horario laboral, uno por hospitalización de un familiar y el cuarto por preferir tratamiento individual. De los 35 que iniciaron tratamiento, abandonaron 3 sujetos, y 3 más, a pesar de realizar más del $80 \%$ del programa, no llegan a completar la evaluación postratamiento, quedando constituido el grupo final por 39 pacientes. Las características socio-demográficas de los 39 sujetos aparecen descritas en la Tabla 2.

\section{Instrumentos de evaluación}

A todas las personas que participaron en el tratamiento se les administró en la primera y última sesión, las siguientes pruebas psicológicas:

Escala de Ansiedad Estado/Rasgo (STAI; Spielberger, Gorsuch y Lushene, 1994). Evalúa el nivel actual de intensidad de la ansiedad, así como la predisposición a responder al estrés. En la versión española la puntuación oscila de 0 a 60 para cada escala, que se traslada a baremos en percentiles o decatipos.

Inventario de Depresión de Beck (BDI; Beck, Ward, Mendelson, Mock y Erbaugh, 1961). Evalúa las manifestaciones conductuales de la depresión en adolescentes y adultos. Contiene un factor cognitivo-afectivo y un factor somático. La puntuación total se obtiene sumando las obtenidas en cada item. Permite clasificar la intensidad de la depresión en: mínima (0-9), leve (10-16), moderada (17-29) y alta $(>30)$.

Body Sensations Questionnaire (BSQ; Chambless, Caputo, Bright y Gallagher, 1984). Valora el miedo a sensaciones físicas asociadas al ataque de pánico en una escala de 5 puntos (desde «nunca me preocupa» hasta «siempre me preocupa»). Consta de 16 items variando las puntuaciones de 0 a 64 . La media de la pun- 
Tabla 2. Características sociodemográficas de los pacientes con TP con o sin agorafobia $(N=39)$

\begin{tabular}{cccc}
\hline & Caracteristicas & Frecuencia & $\%$ \\
\hline Sexo & & & 79,5 \\
Mujer & 31 & 20,5 \\
Hombre & 8 & \\
Estado Civil & & 53,8 \\
Soltero/a & 21 & 38,5 \\
Casado/a & 15 & 5,1 \\
Divorciado/a & 2 & 2,6 \\
Separado/a & 1 &
\end{tabular}

Edad

(en años) media $+/-D T$

$35+/-12,331$

Educación

$\begin{array}{lrr}\text { Analfabeto/a } & 1 & 2,6 \\ \text { Sin estudios } & 1 & 2,6 \\ 1^{\circ} \text { y } 2^{\circ} \text { grado } & 17 & 43,6 \\ 2^{\circ} \text { grado, } 2^{\circ} \text { ciclo } & 14 & 35,9 \\ 3^{\circ} \text { grado(titulados medios) } & 3 & 7,7 \\ 3^{\circ} \text { grado(t. superior) } & 3 & 7,7\end{array}$

Tipo de convivencia

Solo/a

7,7

Con familia propia

Con familia origen

46,2

Solo/a con hijos

2,6

Con otros familiares

Profesión

Profesionales, técnicos/as y similares. 4

Directivos/as y gerentes de Administración Públicas y empresas.

Personal servicios administrativos, empresas y adm. pública.

Comerciantes, vendedores/as y similares

Personal Servicios.

Construcción, industria, minería y trasporte.

Personas con ocupaciones no bien especificadas.

Situación Laboral

Contrato indefinido

Contrato eventual

En paro (no primer empleo)

Estudiante

Labores domésticas

Incapacidad laboral transitoria 
tuación total en población normal es de 1,8 (Bados, 2000).

Agoraphobic Cognition Questionnaire (ACQ; Chambless et al., 1984). Evalúa la frecuencia de pensamientos sobre consecuencias negativas de la ansiedad. Escala de 14 items que se puntúan de 0 a 4 . La puntuación va de 0 a 56.

Cuestionario de Miedos [Fear Questionnaire](FQ; Marks y Mathews, 1979). Versión española de Mathews, Helder y Jonhston (1985). Evalúa la gravedad de tres tipos de fobias (agorafobia, fobia social y fobia a la sangre/inyección/daño) basándose en las conductas de evitación. Evalúa la «fobia total» sumando las 3 subescalas de 5 items. Cada item se evalúa en una escala tipo Likert de 9 puntos $(0=$ «no lo evito» y $8=$ «siempre lo evito»). Las puntuaciones oscilan entre 0 y 120 en fobia total y entre 0 y 40 en cada subescala.

\section{Procedimiento}

El tratamiento se llevó a cabo sobre la base del programa cognitivo-conductual de Barlow (Barlow y Cerny, 1988; Barlow y Craske, 1989) por tratarse de un programa de eficacia probada y por disponer de un manual de autoayuda traducido al castellano (Barlow y Craske, 1993) que sirve de apoyo para el tratamiento.

El núcleo de la teoría cognitivo-conductual es que el pánico está mediado por el miedo a los síntomas de la ansiedad (Barlow, 1988; Clark, 1986; McNally, 1996), en concreto miedo e interpretaciones catastróficas de las sensaciones de pánico (p.ej., me está dando un ataque al corazón, estoy perdiendo la cabeza) o las consecuencias de estas sensaciones (ej: voy a hacer el ridículo, voy a peder el control). Estos pensamientos mantienen alta la ansiedad anticipatoria, facilitando la siguiente crisis de pánico. Con cada crisis, la percepción de peligro se fortalece, lo que fomenta una fuerte respuesta fóbica hacia los propios síntomas. Si se siguen repitiendo, se puede responder ante pequeños signos de arousal (p. ej: una aceleración del latido cardiaco) con miedo condicionado, provocando nuevas crisis. La evitación agorafóbica de situaciones asociadas al pánico ayuda a mantener el trastorno e impide la posible habituación (Gould, Otto y Pollack, 1995).

A continuación resumimos los componentes fundamentales del TCP (según Martin, 2007):

(1) Observación y compresión del trastorno. Este componente pretende establecer las bases para la reestructuración cognitiva. Introduce las diferencias entre las crisis de ansiedad y la ansiedad generalizada, el estrés y el papel que desempeña cada uno en el comienzo y mantenimiento del trastorno. A través de los autoregistros, introduce la observación objetiva de las sensaciones y situaciones que desencadenan las crisis. Su práctica es fundamental para evaluar las recaídas, que por su efecto depresógeno, tienden a desvirtuar los avances realizados. La comprensión del modelo de las crisis de ansiedad y de lo que no puede pasar como consecuencia de la ansiedad, disminuye el miedo lo suficiente como para enfrentarse a la exposición.

(2) Técnicas de control físico: respiración diafragmática y entrenamiento en relajación muscular. No se pretende que estas técnicas se utilicen para reducir o «controlar» las crisis de ansiedad, sino para disminuir el estado general de activación. Es decir, tienen más que ver con la ansiedad generalizada y, en todo caso, detectar señales mínimas de ansiedad para poder reducirlas. Digamos que tienen una finalidad más preventiva y que sólo surten efecto si se practican de forma regular (y no cuando se sufre una crisis).

(3) Reestructuración cognitiva: identificar las distorsiones cognitivas que acompañan al trastorno de pánico y reevaluarlas de manera que se contraste el peligro real. Una pregunta típica de esta fase puede ser: ¿Cuál es la probabilidad de que te de un infarto? Esta pregunta supone: haber identificado ese pensamiento, comprender lo que es un infarto y haber buscado información fiable.

(4) Exposición interoceptiva: quizás el componente más novedoso, supone buscar las sensaciones en lugar de tratar de evitarlas o reducirlas. Se comienza provocando sensaciones de ansiedad en un ambiente controlado, mediante ejercicios diseñados específicamente como hiperventilar o girar en una silla. Después se pide al paciente que lleve a cabo estos ejercicios sólo 
y finalmente que busque situaciones que provocan sensaciones similares a las que teme. E1 resultado que se persigue es la extinción de la falsa alarma (crisis de ansiedad)

(5) Exposición: se dirige a disminuir o, si fuera posible, eliminar la agorafobia. Estrictamente hablando, no sería necesario utilizarla en el trastorno de pánico sin agorafobia. Su objetivo es impedir las conductas de escape y de evitación que mantienen o agravan el trastorno. La exposición debe ser sistemática y regular, independientemente del estado de ansiedad de un día concreto.

El tratamiento se llevó a cabo de acuerdo con el siguiente protocolo:

1. Comienzo del grupo y recogida de datos. Dos profesionales de salud mental: una psicóloga clínica (coautora de este trabajo), y una coterapéuta enfermera especialista en salud mental, llevaron a cabo el tratamiento. Ambas están entrenadas para aplicar este programa. El TCP se aplica en 12 sesiones grupales semanales de 90 minutos en el Centro de Salud Mental. La primera y la última son resúmenes pre/ postratamiento y administración de escalas. El grupo comienza cuando aceptan participar entre 8 y 12 pacientes, después de informarles brevemente de la duración y frecuencia de las sesiones y de los objetivos del tratamiento. Se recogen los datos de filiación de la historia clínica: sexo, fecha nacimiento, nivel educativo, estado civil, profesión, situación laboral. También el diagnóstico principal y diagnóstico secundario si lo hay (comorbilidad). La enfermera llama a los pacientes en lista de espera para grupo y les informa del día y hora de la primera sesión. La mayoría ya han iniciado o continúan el tratamiento farmacológico.

2. Primera sesión grupal: evaluación y explicación del modelo cognitivo del pánico. Se siguió la Guía para el tratamiento grupal del trastorno de pánico (Martín, 2007). Tras constatar que el TP, con o sin agorafobia, es el principal problema de los componentes del grupo, se realiza una evaluación pre- y post de las siguientes variables: ansiedad, depresión, temor a las sensaciones de pánico, cogniciones relacionadas con el pánico, agorafobia y otras fobias y medicación al inicio del tratamiento. Para la evaluación pre- y postratamiento, se utiliza- ron los instrumentos descritos atrás. Por último se les recomienda la lectura del manual de Barlow y Craske (1993) Domine la ansiedad y el pánico.

3. Aplicación de la terapia de grupo. Sesiones intermedias. Se dividió en las siguientes etapas: (1) Explicación y compresión del trastorno y del modelo cognitivo del pánico. (2) Manejo de la ansiedad fisiológica; técnicas de control físico. (3) Reestructuración cognitiva. (4) Experimentos conductuales; exposición interoceptiva. (5) Eliminación de cualquier conducta evitativa de situaciones y actividades y exposición a las mismas. Y (6) prevención de recaídas.

Cualquier tarea o aprendizaje de técnica se consideró como un experimento para poner a prueba la hipótesis catastrofista propia de estos pacientes, frente a la hipótesis alternativa propuesta por el modelo del control del pánico. A lo largo de toda la terapia se hizo un esfuerzo por mantener el estilo socrático, formulando preguntas dirigidas a que el propio sujeto «descubra» las conclusiones a que queremos hacerle llegar. En todo momento planteamos (y habituamos al paciente a plantear) sus creencias catastrofistas como hipótesis a verificar, instándole a considerar del mismo modo la explicación que ofrece el modelo cognitivo: como hipótesis alternativa para la cual también se buscan pruebas objetivas.

Como tareas para casa los pacientes fueron leyendo los capítulos del libro de autoayuda trabajados en cada sesión (Barlow y Craske et al., 1993) y cumplimentan los registros de sensaciones temidas, interpretaciones catastrofistas, práctica de respiración diafragmática y relajación muscular y «exposición». Estas tareas se revisan y actualizan en cada sesión.

Al finalizar de las doce sesiones se realiza la evaluación postratamiento. Se concluye de forma personalizada decidiendo el tratamiento recomendable o el alta terapéutica hasta la sesión de seguimiento a los 3 meses.

\section{RESULTADOS}

Para el análisis descriptivo de las variables sociodemográficas de los participantes, de la 
duración del trastorno y el seguimiento del tratamiento farmacológico se calcularon medias, desviaciones típicas y porcentajes. Se compararon los resultados en las escalas citadas entre pre- y postratamiento utilizando la prueba $t$ de Student. El intervalo de confianza usado fue del 95\%. Todos los análisis estadísticos fueron realizados utilizando SPSS versión 15.0.

Los datos sociodemográficos, que presentados en la Tabla 2, nos muestran un perfil tipo de paciente que acude al Centro de Salud Mental con diagnóstico de TP: mujer de 35 años, soltera, con nivel de estudios alcanzados de $2^{\circ}$ grado, que vive con su familia de origen, con una ocupación no bien especificada, bien con contrato indefinido o como estudiante. Dicho perfil coincide con las investigaciones de la literatura científica (Furukawa, Watanabe y Churchill, 2006; Mitte, 2005; Gould et al., 1995).

Respecto a la gravedad del trastorno, los años trascurridos desde la primera crisis de pánico hasta el inicio del grupo de tratamiento oscilaron entre 15 días y 27 años y 3 meses (media $=5,76, D T=7,06) . \mathrm{Al}$ inicio del grupo el $89 \%$ de los 39 pacientes seguían tratamiento psicofarmacológico (de los cuales el 79,5\% ya habían seguido otros tratamientos farmacológicos anteriormente), el 3\% no respondía al tratamiento farmacológico y el $8 \%$ restante estaba libre de cualquier tipo de tratamiento farmacológico.

Tabla 3. Medias y DTs (entre paréntesis) durante el pre- y el postratamiento $(N=29)$

\begin{tabular}{lccc}
\hline & Pre-tratamiento & Postratamiento & $t(28)$ \\
\hline $\begin{array}{l}\text { Depresión (BDI) } \\
\text { Miedos (FQ): }\end{array} \quad 18,72(9,32)$ & $8,86(7,32)$ & $8,25^{* * *}$ \\
$\quad$ Total & $49,45(22,59)$ & $30,66(21,62)$ & $5,49^{* * *}$ \\
$\quad$ Agorafobia & $19,14(11,65)$ & $10,59(9,84)$ & $4,53^{* * *}$ \\
$\quad$ Sangre & $13,62(9,32)$ & $7,93(9,07)$ & $4,65^{* * *}$ \\
$\quad 16,34(9,65)$ & $12,00(19,72)$ & $3,32^{* *}$ \\
$\quad$ Fobia social & $35,45(14,74)$ & $24,28(12,88)$ & $4,41^{* * *}$ \\
Sensaciones corporales (BSQ) & $23,03(10,95)$ & $13,28(9,56)$ & $4,71^{* * *}$ \\
Cogniciones agorafóbicas (ACQ) & & & $6,76^{* * *}$ \\
Ansiedad (STAI): & $69,79(20,96)$ & $38,69(28,72)$ & $6,46^{* * *}$ \\
$\quad$ Estado de ansiedad & $86,52(13,62)$ & $55,03(28,93)$ & \\
$\quad$ Rasgo de ansiedad & & & \\
\hline
\end{tabular}

Nota. Los valores de las dos variables de ansiedad se indican en percentiles. BDI = Inventario de Depresión de Beck; FQ = Fear Questionnaire; BSQ = Body Sensations Questionnaire; ACQ = Agoraphobic Cognition Questionnaire. STAI = State-Trait Anxiety Inventory. ${ }^{* *} p<0,01 ; * * * p<0,001$.

Los estadísticos descriptivos de la muestra en las diferentes variables psicológicas, en el pre y postratamiento, se muestran en la Tabla 3. Los sujetos al inicio del tratamiento psicológico presentaban TP de gravedad severa y de tipo crónico. El 79,5\% seguía tratamiento farmacológico desde hacía años. La puntuación media en el BDI fue de 18,72 (depresión moderada). La ansiedad estado era alta (percentil 69,79 del STAI-E) y la ansiedad rasgo muy alta (percentil 86,52 del STAI-R). En el índice de miedos general la puntuación media (PM) fue 3,2, que se sitúa en el intervalo entre «lo evito poco» a «decididamente lo evito»; igual que las subescalas agorafobia y fobia social $(\mathrm{PM}=3,8$ y 3,3 respectivamente). En la subescala de sangre/inyecciones/daño la PM fue de 2,7 («lo evito poco»). La respuesta media en cogniciones agorafóbicas fue de 1,6 (los pensamientos se presentan «la mitad de las veces») y en sensaciones fisiológicas propias de la ansiedad fue de 2,21 superando el 1,8 considerado punto de corte de la normalidad (Bados, 2000).

En la Tabla 3 presentamos también los resultados de los análisis de las diferencias prepost basados en la $t$ de Student. Se encontraron diferencias significativas entre el pre y el pos- 
tratamiento en todas las respuestas evaluadas. Las medias postratamiento en Ansiedad Estado (centil: 38,69) y Ansiedad Rasgo (centil: 55,03 ) disminuyeron hasta niveles considerados dentro de la normalidad. Se produce una reducción significativa de conductas evitativas, presentado en la escala de miedos total una respuesta media de 2,04 $(\mathrm{PD}=30,66)$. En la subescala de Agorafobia se reduce hasta 2,11 $(\mathrm{PD}=10,59)$ y en la subescala de fobia social a 2,4 $(\mathrm{PD}=12)$. Todas corresponden a «lo evito poco». En la subescala de sangre/ inyecciones/ daño puntúan 1,5 (entre «no lo evito» o «lo evito poco»). El miedo a las sensaciones fisiológicas se reduce hasta una respuesta media de 1,51 $(\mathrm{PD}=24,28)$ que corresponde a «algunas veces» y el nivel de creencias erróneas agorafóbicas desciende hasta $0,94(\mathrm{PD}=$ $13,28)$ predominando la respuesta «nunca o casi nunca». Los resultados postratamiento son congruentes con los datos recogidos sobre el seguimiento del tratamiento farmacológico (véase la Figura 1). Al final del tratamiento de los 29 sujetos, 6 no tomaban ningún fármaco (tres coinciden con los que no tomaban tratamiento al inicio) y 15 de los 23 restantes habían disminuido la dosis. Seis mantenían la dosis inicial y ninguno necesitó aumentarla durante el tratamiento grupal.

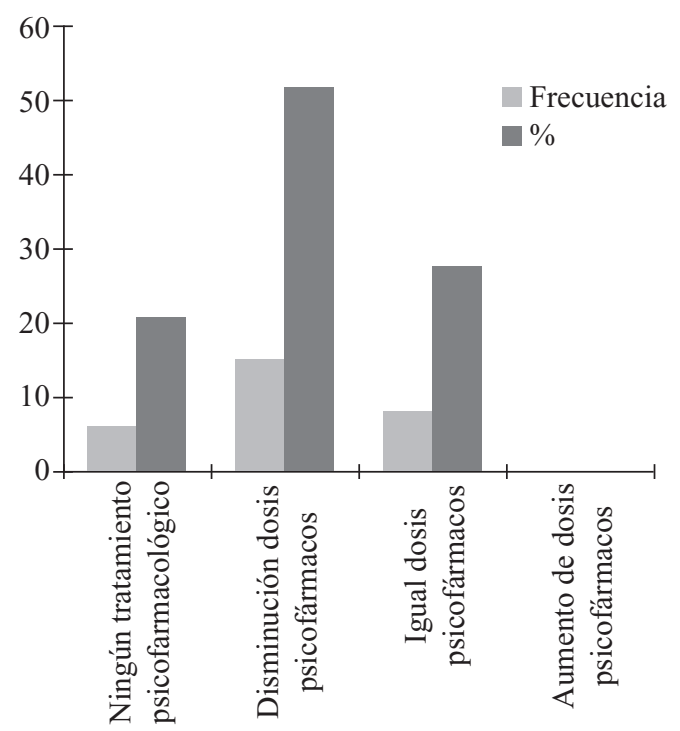

Figura 1. Cambios farmacológicos durante tratamiento $(N=29)$

\section{DISCUSIÓN}

Los resultados obtenidos avalan la efectividad de la terapia de control del pánico de Barlow y Cerny (1988) aplicada en formato grupal a pacientes con TP con o sin agorafobia en un servicio público de Salud Mental.

Se trata simultáneamente entre 8 y 12 pacientes con trastorno de pánico de larga evolución, con sintomatología asociada agorafóbica y depresiva, resistentes al tratamiento farmacológico en, prácticamente, 10 sesiones. Respecto al tratamiento individual, mejora la relación coste-beneficio y ofrece un tratamiento de calidad de una sesión semanal, con mejora significativa en todas las áreas evaluadas, a pesar de la gravedad de los casos.

De los 35 pacientes que iniciaron el tratamiento sólo abandonaron tres. Supone un índice de abandono del 8,6\%, situándose en la parte baja de los estudios de efectividad (Tabla 1) y mejorando los obtenidos en otros estudios: $12.7 \%(D T=10,92)$ en TCC, $15,11 \%(D T=$ $12,83)$ en terapia conductual, 20,4\% $(D T=$ $15,27)$ en farmacoterapia y $20,5 \%(D T=11,18)$ en tratamiento combinado (Mitte, 2005). Los 32 pacientes restantes mantuvieron un nivel de adherencia elevado al asistir a más del $80 \%$ de las sesiones.

Se producen ganancias significativas en todas las dimensiones relacionadas con el TP con o sin agorafobia, con diferencias medias de ansiedad estado (STAI-E) de 31,10 (IC 95\% 21,67 a 40,52, $p<0,001)$, síntomas fisiológicos de las crisis de pánico (BSQ) de 11,17 (IC 95\% $=6,01$ a 16,32, $p<0,001)$, conductas agorafóbicas de 8,55 (IC 95\% = 4,68 a 12,41, $p<$ $0,001)$ y creencias agorafóbicas (ACQ) de 9,75 $($ IC $95 \%=5,51$ a $14,00, P=p<0,001)$.

También mejoran escalas que no eran objeto específico del tratamiento, como la depresión (BDI) con una diferencia media de 9,862 (IC $95 \%=7,41$ a $12,31, p<0,001)$ o la ansiedad rasgo (STAI-R) con una diferencia media de $31,48($ IC $95 \%=21,50$ a $41,46, p<0,001)$ llegando a puntuar dentro de la normalidad. Entendemos que la cronicidad de los cuadros de TP de bastantes pacientes había empeorado su calidad de vida provocando sentimientos de desesperanza, impotencia y baja autoestima. 
Todo esto configura un cuadro asociado de tipo depresivo y un estado general de ansiedad entre crisis que tiende a cronificar el TP. La mejoría de los síntomas de pánico, la eliminación de las conductas evitativas y, en consecuencia, la mejoría en calidad de vida, parece disminuir hasta normalizar los síntomas depresivos y la ansiedad generalizada.

El entrenamiento en respiración y relajación, junto con la psicoeducación acerca de los síntomas fisiológicos de la ansiedad, ayudaría a los pacientes a generalizar estos aprendizajes a otras situaciones ansiógenas, lo que justificaría la mejoría encontrada en la subescala de fobia social y miedo a sangre-inyecciones-daño, con una diferencia media respectivamente de 4,34 $(I C 95 \%=1,66$ a $7,02, p<0,01)$ y 5,69 (IC $95 \%=3,18$ a 8,$19 ; p<0,001)$.

Comparado con otros estudios de efectividad realizados en grupo, tanto en España como en otros países el programa produce reducciones significativas en las escalas coincidentes (Wade et al., 1998; Galassi et al., 2007; GarcíaPalacios et al., 2002). También resiste la com- paración con los dos estudios de eficacia realizados de forma individual o grupal que consideramos como benchmarking (Clark, et al., 1994; Telch, et al., 1993) (véase la Tabla 4).

Aunque intentamos recoger la duración exacta del tratamiento farmacológico previo no fue posible, en la mayoría de los casos por olvido de los pacientes, por lo que renunciamos al análisis de este dato. Varios de los estudios citados mantienen una pauta constante de medicación a lo largo de TCC grupal. El 89,7\% de los pacientes seguían tratamiento farmacológico al inicio del grupo y el $79 \%$ ya habían tomado anteriormente otros tipos de psicofármacos. La duración media de los trastornos es de 5 años y medio, y un alto porcentaje de pacientes seguía más de un tratamiento farmacológico. Observamos la pauta de medicación que había seguido el psiquiatra al finalizar el grupo: del total, 6 terminan sin medicación (tres más que al inicio: 20,7\%), 15 disminuyen la dosis $(51,7 \%), 8$ mantienen la misma dosis $(27,6 \%)$ y ninguno habían necesitado aumentarla $(0 \%)$. Podemos considerar que la causa de la mejoría

Tabla 4. Medidas de resultado en estudios similares. Medias y DTs (entre paréntesis)

\begin{tabular}{|c|c|c|c|c|c|c|c|c|c|c|c|c|}
\hline & \multicolumn{2}{|c|}{$\begin{array}{c}\text { Garriga, } \\
\text { Martín y López- } \\
\text { Soler (este } \\
\text { estudio) }\end{array}$} & \multicolumn{2}{|c|}{$\begin{array}{l}\text { Wade et al. } \\
\text { (1998) }\end{array}$} & \multicolumn{2}{|c|}{$\begin{array}{c}\text { García- } \\
\text { Palacios et al. } \\
\text { (2002) }\end{array}$} & \multicolumn{2}{|c|}{$\begin{array}{c}\text { Galassi et al. } \\
\text { (2007) }\end{array}$} & \multicolumn{2}{|c|}{$\begin{array}{l}\text { Telch et al. } \\
\text { (1993) }\end{array}$} & \multicolumn{2}{|c|}{$\begin{array}{c}\text { Clark et al. } \\
\text { (1994) }\end{array}$} \\
\hline & Pre- & Post- & Pre- & Post- & Pre- & Post- & Pre- & Post- & Pre- & Post- & Pre- & Post- \\
\hline Agorafobia & $\begin{array}{c}19,14 \\
(11,66)\end{array}$ & $\begin{array}{l}10,59 \\
(9,84)\end{array}$ & $\begin{array}{c}16,5 \\
(10,8)\end{array}$ & $\begin{array}{c}8,8 \\
(6,9)\end{array}$ & $\begin{array}{c}19,25 \\
(11,7)\end{array}$ & $\begin{array}{c}10,37 \\
(10,7)\end{array}$ & & & $\begin{array}{c}12,18 \\
(11,41)\end{array}$ & $\begin{array}{c}5,06 \\
(6,76)\end{array}$ & $\begin{array}{l}13,4 \\
(5,7)\end{array}$ & $\begin{array}{c}8,3 \\
(4,0)\end{array}$ \\
\hline Sangre & $\begin{array}{l}13,62 \\
(9,33)\end{array}$ & $\begin{array}{c}7,93 \\
(9,07)\end{array}$ & $\begin{array}{l}14,9 \\
(8,2)\end{array}$ & $\begin{array}{l}10,7 \\
(7,1)\end{array}$ & & & & & & & $\begin{array}{l}12,6 \\
(4,5)\end{array}$ & $\begin{array}{l}10,0 \\
(3,5)\end{array}$ \\
\hline Fobia social & $\begin{array}{l}16,34 \\
(9,66)\end{array}$ & $\begin{array}{c}12,00 \\
(19,72)\end{array}$ & $\begin{array}{l}17,3 \\
(8,9)\end{array}$ & $\begin{array}{l}10,1 \\
(6,5)\end{array}$ & & & & & & & $\begin{array}{l}11,1 \\
(5,1)\end{array}$ & $\begin{array}{c}8,2 \\
(3,6)\end{array}$ \\
\hline BSQ & $\begin{array}{c}35,45 \\
(14,74)\end{array}$ & $\begin{array}{c}24,28 \\
(12,88)\end{array}$ & & & & & & & $\begin{array}{l}33,74 * \\
(11,15)\end{array}$ & $\begin{array}{r}13,94 * \\
(8,52)\end{array}$ & $\begin{array}{l}38,6,4 \\
(9,5)\end{array}$ & $\begin{array}{l}22,5 \\
(5,2)\end{array}$ \\
\hline ACQ & $\begin{array}{c}23,03 \\
(10,96)\end{array}$ & $\begin{array}{l}13,28 \\
(9,56)\end{array}$ & & & & & & & & & $\begin{array}{l}27,9 \\
(6,5)\end{array}$ & $\begin{array}{l}19,4 \\
(3,3)\end{array}$ \\
\hline STAI-Estado & $\begin{array}{c}69,79 \\
(20,93)\end{array}$ & $\begin{array}{c}38,69 \\
(28,72)\end{array}$ & & & & & $\begin{array}{c}49,56 \\
(10,43)\end{array}$ & $\begin{array}{l}42,24 \\
(9,08)\end{array}$ & & & & \\
\hline STAI-Rasgo & $\begin{array}{c}86,52 \\
(13,62)\end{array}$ & $\begin{array}{c}55,03 \\
(28,93)\end{array}$ & & & & & $\begin{array}{c}54,12 \\
(11,51)\end{array}$ & $\begin{array}{c}45,97 \\
(11,47)\end{array}$ & & & & \\
\hline BDI & $\begin{array}{l}18,72 \\
(9,33)\end{array}$ & $\begin{array}{c}8,86 \\
(7,33)\end{array}$ & $\begin{array}{l}15,5 \\
(8,8)\end{array}$ & $\begin{array}{c}6,0 \\
(6,3)\end{array}$ & $\begin{array}{l}15,54 \\
(9,54)\end{array}$ & $\begin{array}{c}6,36 \\
(4,57)\end{array}$ & $\begin{array}{l}16,00 \\
(8,54)\end{array}$ & $\begin{array}{l}10,24 \\
(7,19)\end{array}$ & $\begin{array}{l}16,85 \\
(8,21)\end{array}$ & $\begin{array}{c}7,68 \\
(5,27)\end{array}$ & $\begin{array}{l}17,0 \\
(7,8)\end{array}$ & $\begin{array}{c}6,3 \\
(6,4)\end{array}$ \\
\hline
\end{tabular}

Nota . BDI = Inventario de Depresión de Beck; BSQ = Body Sensations Questionnaire; ACQ = Agoraphobic Cognition Questionnaire. STAI = State-Trait Anxiety Inventory. 
es fundamentalmente la Terapia de Control del Pánico grupal de Barlow (1988).

Los resultados permiten recomendar este modelo para el tratamiento de los TP, con o sin agorafobia, en una consulta pública de salud mental, basándonos en su efectividad o utilidad clínica (Eje II de la Template for Developing Guidelines: Interventions for Mental Disorders and Psychosocial Aspects of Physical Disorders; de la Task Force on Psychological Interventions Guidelines, APA, 1995). La dinámica de trabajo actual de los CSM públicos de la región de Murcia, reflejada en este estudio, pone de manifiesto que el tratamiento cognitivo-conductual no es considerado en el Sistema Regional de Salud como primera elección en el tratamiento del TP con o sin agorafobia, en contra de las recomendaciones de las guías de práctica clínica de alta calidad, del National Institute for Health and Clinical Excellence (NICE, 2004; 2007) y de la NHS Economic Evaluation Database (NHS EED) sobre mejor alternativa coste-beneficio. Normalmente es la tercera opción, después de médico de familia y psiquiatra. La derivación al tratamiento psicológico se hace con una media de 5 años de retraso.

Aunque no hemos valorado cuantitativamente el impacto de los grupos para las listas de espera, la experiencia de trabajo y la impresión clínica indica que facilita el acceso a un tratamiento adecuado a mayor número de personas en menor tiempo. El tiempo de espera va de 0 días a 10-11 semanas como máximo (tiempo en el que acaba el grupo iniciado). Si en la atención individual los pacientes pueden estar en lista de espera para psicología de 6 a 12 meses, las terapias grupales, pueden reducir estos tiempos de forma notable. Además, si hay un número suficiente de pacientes, podría iniciar tratamiento un segundo grupo antes de la finalización del primero, lo que reduciría la espera.

El índice de satisfacción general es muy alto, según manifestaciones espontáneas de los pacientes. Todo ello nos permite señalar la necesidad de formar en tratamientos grupales a los profesionales de salud mental y a los psicólogos internos residentes. Los tratamientos grupales de elección deberán cumplir los criterios de excelencia clínica, incluir manuales para el terapeuta que aseguren una práctica correcta y, a ser posible, un manual de autoayuda para los pacientes.

Respecto a las posibilidades de mejora de este trabajo señalar que: (1) Hubiera sido deseable un estudio de seguimiento para evaluar el mantenimiento o no de la mejoría, y si las pautas de medicación siguen en descenso. En la práctica ambulatoria pública el seguimiento resulta complicado. La consulta suele estar completa los meses siguientes y los espacios grupales ocupados por nuevos grupos debido a la alta presión asistencial. Muchos pacientes piden permiso en su trabajo para acudir semanalmente al centro. Cuando acaban el tratamiento suelen tener problemas para volver, sobre todo si se mantiene la mejoría y viven el TP como algo ya superado. (2) Consideramos necesario introducir una entrevista psicodiagnóstica estructurada para mejorar la calidad del diagnóstico, así como evaluadores independientes. (3) Aunque en las entrevistas clínicas previas al inicio del grupo se pregunta sobre la frecuencia de las crisis de pánico, no hemos podido contar con este dato en muchos casos. Dado que la evaluación estandarizada en TP recomienda incluirla, sería un área de mejora para futuras investigaciones. También es conveniente aplicar escalas como el Indice de Sensibilidad a la Ansiedad (ASI) o la Escala de gravedad para el trastorno de pánico (PDSS) de las que existe versión castellana (Sandín, Valiente, Chorot y Santed, 2007; Bulbena y Martín Santos, 1992) y que se utilizan en estudios nacionales e internacionales. (4) Gran parte de los pacientes derivados al grupo ya habían iniciado tratamiento con psicofármacos ansiolíticos o antidepresivos y no podemos valorar el alcance de este modelo de TCC grupal sin medicación. Sería conveniente ofrecer la posibilidad de este tipo de tratamiento, en colaboración con los médicos de familia o los servicios de urgencias, lo antes posible tras la detección del trastorno: el beneficio puede ser mayor, se evita cronicidad y complicaciones concomitantes y disminuyen las recaídas. El paciente, mediante el consentimiento informado, podría decidir qué tratamiento prefiere. Y (5) no hemos podido contar con un grupo control de sólo trata- 
miento farmacológico o en lista de espera que permitiera eliminar sesgos, por lo que hemos utilizado una estrategia de benchmarking. En futuras investigaciones puede contemplarse un grupo control que mejore la calidad metodológica.

\section{REFERENCIAS}

American Psychiatric Association (1994). Diagnostic and statistical manual of mental disorders (4ed.). Washington, DC: APA (Barcelona Masson, 1994)

American Psychiatric Association. Task Force on Promotion and Dissemination of Psychological Procedures (1995). A report to the Division 12 board of the American Psychological Association. (available from the Division 12 of the American Psychological Association), 750, first street, NE, Washington

American Psychiatric Association. Work Group on Panic Disorder (1998). Practice guideline for the treatment of patients with panic disorder. American J. Psychiatry 155, 1-34.

Bados, A. (2000). Agorafobia y ataques de pánico. Madrid. Pirámide.

Barlow, D.H. (1988). Anxiety and its disorders: The nature and treatment of anxiety and panic. New York: Guilford.

Ballester, R., y Botella, C. (1992). Aplicación del programa cognitivo comportamental de Clark y Salkovskis en grupo: Una alternativa para el tratamiento del pánico. Análisis y Modificación de Conducta, 18, 291-321

Barlow, D.H., y Cerny, J.A. (1988). Psychological treatment of panic. New York: Guilford Press.

Barlow, D.H., y Craske, M.G. (1989). Mastery of your anxiety and panic. Albary. NY: Graywind.

Barlow, D.H., y Craske, M.G. (1994). Mastery of your anxiety and panic. II. San Antonio, TX: The Psychological Corporation.

Barlow, D.H., y Craske, M.G. (1993). Domine la ansiedad y el pánico. Traducción y edición de Julio C. Martín García-Sancho. Murcia.

Barlow, D. H., Gorman, J. M., Shear, M. K., y Woods, S. W. (2000). Cognitive-behavioral therapy, imipramine, or their combination for panic disorder. A randomized controlled trial. JAMA, 283, 2529-2536.

Barlow, D.H., DiNardo, P.A., Vermilyea, B.B., y Blanchard, E.B. (1986). Co-morbidity and depression among the anxiety disorders, Issues in classification and diagnosis, Journal. Nervous and Mental Disease, 174, 63-72.

Beck, A.T., Ward, C.H., Mendelson, M., Mock, J.E., y Erbaugh, T. (1961). A inventory for measuring depression. Archives of General Psychiatry, 4, 53-63.
Beck, J.G., y Zebb, B.J. (1994). Behavioral assessment and treatment of panic disorder: Current status, future directions. Behavior Therapy, 25, 581-611.

Belfer, P.L., Muñoz, L.S., Schachter, J., y Levendusky, P.G. (1995). Copgnitive-Behavioral group psychotherapy for agoraphobia and panic disorder. International Journal of Group Psychotherapy. 45, 185-206.

Botella, C. (2001). Tratamientos psicológicos eficaces para el trastorno de pánico. Psicothema, 13, 465-478

Botella, C. (2004). Tratamientos eficaces para el trastorno de pánico. En M. Pérez Álvarez, J.R. Fernández Hermida, C. Fernández Rodríguez e I. Amigó (Eds.), Guía de Tratamientos Psicológicos Eficaces I. Madrid: Pirámide.

Brown, T.A., y Barlow, D.H. (1992). Panic disorder and panic disorder with agoraphobia. En P.H. Wilson (Ed.), Principles and practice of relapse prevention (pp. 191212). New York: Guilford Press.

Bulbena, A., y Martín Santos, R. (1992). Escala de Gravedad del Trastorno de Pánico. Departamento de Psiquiatría, Facultad de Medicina. Universidad de Barcelona (policopiado)

Chambless, D.L., Caputo, G.C., Bright, P., Gallagher, R. (1984). Assessment of fear of fear in agoraphobics: the body sensations questionnaire and the agoraphobic cognitions questionnaire. Journal Consulting Clinical Psychology. 52, 1090-7.

Clark, D.M. (1986). A cognitive approach to panic. Behavioral Research and Therapy, 24, 461-470.

Clark, D.M. (1989). Anxiety States: Panic and generalized anxiety. En K. Hawton, P.M. Slaskovskis, J. Kirk y D.M. Clark (Eds.), Cognitive behaviour therapy for psychiatric problems: A practical guide (pp. 52-96) Oxford University Press.

Clark, D.M., Slaskovskis, J., Hackmann, A., Middleton, H., Anastasiades, P., y Helder, M. (1994). A comparison of cognitive therapy, applied relaxation and imipraminee in the treatment of panic disorder. British Journal of Psychiatry. 164, 759-769.

Clum, G.A., y Surls, R. (1993); A meta-analysis of treatments for panic disorder. Journal Consulting Clinical Psychology, 61, 317-326

Craske, M.G., Brown, T.A., y Barlow, D.H. (1991). Behavioral treatment of panic: A two years follow-up. Behavior Therapy, 22, 289-304.

Furukawa, T.A., Watanabe, N., y Churchill, R. (2006). Psychotherapy plus antidepressant for panic disorder with or without agoraphobia. British Journal of Psychiatry. 188, 305-312.

Galassi, F., Quercioli, S., Carismas, D., Niccolai, V., y Barciulli, E. (2007). Cognitive-Behavioral group treatment for panic disorder with agoraphobia. Journal Clinical Psychology, 63, 409-416.

García-Palacios, A., Botella, C., Roberts, C., Baños, R., Perpiñá, C., Quero, S., y Ballester, R. (2002). Clinical Utility of Cognitive-Behavioural Treatment for Panic 
Disorders. Results obtain in Different Settings: A Research Centre and a Public mental Health Care Unit. Clinical Psychology and Psycotherapy, 9, 373-383.

Gould, R.A., Otto, M.W., Pollack, M.H. (1995). A metaanalysis of treatment outcome for panic disorder. Clinical Psychology Review, 15, 819-844.

Jacobson, N.S., y Revenstorf, D. (1988). Statistics for assessing the clinical significance of psychotherapy techniques: Issues, problems and new developments. Behavioral Assessment, 10, 133-145.

Keller, M.B., Yonkers, K.A., Warshaw, M.G., Pratt, L.A. Golan, J., Mathews, A.O., White, K., Swartz, A., Reich, J., y Lavori, P. (1994). Remission and relapse in subjects with panic disorder and panic with agoraphobia: a prospective short interval naturalistic follow-up. Journal Nervous Mental Disorders, 182, 290-296.

Klerman, G., Weissman, M.M., Oullette, R. Johnson, J., y Greenwald, S. (1991). Panic attacks in the community social morbidity and health care utilization, Journal American Medical Association, 265, 742-746.

Leveni, D., Mazzoleni, D., y Piancentini, D. (1999). Cognitive-Behavioral group treatment of panic attacks disorder: A description of the results obtained in a public mental health service. Epidemiologia e Psichiatria Sociale, 8, 270-275.

Lidren, D.M., Watkins, P.L., Gould, R.A., Clum, G.A., Asterino, M., y Tulloch, H.L. (1994). A comparison of bibliotherapy and group therapy in the treatment of panic disorder. Journal of Consulting and Clinical Psychology, 62, 865-869.

Marchand, A., Germain, V., Reinharz, D., Mainguy, N., y Landry, P. (2004). Analysis of the cost and effectiveness of a psychotherapy for panic disorder with agoraphobia (PDA) versus a treatment combining pharmacotherapy and psychotherapy. NHS Economic Evaluation Database (NHS EED). Centre for Reviews and Dissemination. University of York.

Margraf, J., Barlow, D.H., Clark, D., y Telch, M.J. (1993). Psychological treatment of panic: work in progress on outcome, active ingredients and follow-up. Behavior Research and Therapy, 31, 1-8.

Markowitz, J.S., Weissman, M.M., Oullette, R., Lish, J.D., y Klerman, G.L. (1989). Quality of life in panic disorder. Archives of General Psychiatry, 46, 984-992.

Marks, I.M. (1987). Fear, phobias and rituals. New York: Oxford University Press.

Marks, I.M., y Mathews, A.M. (1979). Brief standard self-rating scale for phobic patients. Behaviour Research and Therapy, 17, 263-267.

Martin Garcia-Sancho, J.C. (2007). Guía para el tratamiento grupal del trastorno de pánico. En Moreno, P., y Martín J.C. (Ed.), Tratamiento psicológico del trastorno del pánico y la agorafobia. Manual para terapeutas. Bilbao: Desclée.de Brouwer, Nuevas psicoterapias. Biblioteca de Psicología.
Martisen, E.W., Olsen, T., Tonset, E., Nyland, K.E., y Aarre, T.F. (1998). Cognitive-behavioral group therapy for panic disorder in the general clinical setting: A naturalistic study with 1-year follow-up. Journal of Cognitive Psychotherapy, 59, 437-442.

Mathew, A.M., Gelder, M.G., y Johnson, D.W. (1985). Agorafobia Naturaleza y Tratamiento. Barcelona: Editorial Fontanella.

Mavissakalian, M. (1990). The relationship between panic disorder agoraphobia and personality disorders. Psychiatric Clinical North America, 13, 661-684.

McNally, R.J. (1996). Nuevos desarrollos en el tratamiento del trastorno de pánico. Revista de Psicopatología y Psicología Clínica, 1, 91-103.

Mitte, K. (2005). A meta-analysis of the efficacy of psycho- and pharmacotherapy in panic disorder with and without agoraphobia. Journal of Affective Disorders, $88,27-45$.

Michelson, L.K., y Marchione, K. (1991). Behavioral, cognitive and psychophysiological treatments of panic disorder with agoraphobia: Critique and synthesis. Journal Consulting Clinical Psychology, 59, 100-114.

Natahn, P.E., y Gorman, J.M. (1998). A guide to treatments that work. Oxford: Oxford University Press.

National Institute for Clinical Excellence NICE (2004). Clinical guideline, 22. December. London.

National Institute for Clinical Guideline 22 (2007). Anxiety. Management of anxiety (panic disorder, with or without agoraphobia, and generalised anxiety disorder) in adults in primary, secondary and community care. NICE clinical guideline 22 (2007) Available from www.nice.org.uk/CG022.

National Institute of Health. NIH (1991). Consensus Development Conference Statement, Vol 9, $\mathrm{n}^{\circ}$ 2, Treatment of Panic Disorder, Bethesde, MD: NIH

Organización Mundial de la Salud (1992). CIE-10. Clasificación de los trastornos mentales y del comportamiento. Madrid: Meditor.

Ost, L.G., Westling, B.E., y Hellstrom, K. (1993). Applied relaxation, exposure in vivo and cognitive methods in the treatment of panic disorder with agoraphobia. $\mathrm{Be}$ havior Research and Therapy, 31, 383-394.

Penava, S.J., Otto, M.W., Maki, K.M., y Pollack, M.H. (1998). Rate of improvement during cognitive-behavioral group treatment for panic disorder. Behaviour Research and Therapy, 36, 665-673.

Pollack, M.H., Otto, M.W., Rosenbaum, J.F., y Sachs, G.S. (1992). Personality disorders in patients with panic disorders: association with panic childhood anxiety disorders, early trauma, comorbidity and chronicity. Comprehensive Psychiatry, 33, 78-83.

Robins, L.N., Helzer J.E., Weissman M., Orvaschel H., Grunberg, E., Burke, J.D., y Regier, D.A. (1984). Lifetime prevalence of specific psychiatric disorders in three sites. Archives of General Psychiatry, 41, 949-958. 
Sanderson, W.S., DiNardo, P.A., Rappe, R.P., y Barlow, D.H. (1990). Syndrome comorbidity in patients diagnosed with a DSM-IIIR anxiety disorder. Journal $A b$ normal Psychology, 99, 308-312.

Sandín, B., Valiente, R.M., Chorot, P., y Santed, M.A. (2007). ASI-3: Nueva escala para la evaluación de la sensibilidad a la ansiedad. Revista de Psicopatología y Psicología Clínica, 12, 91-104.

Sandín, B., Rodero, B., Santed, M.A., y García-Campayo, J. (2006). Sucesos vitales estresantes y trastorno de pánico: relación con la ocurrencia del trastorno de pánico, la gravedad clínica y la agorafobia. Revista de Psicopatología y Psicología Clínica, 11, 179-190.

Sharp, D. M., Power, K. G., y Swanson, V. (2004). A comparison of the efficacy and acceptability of group versus individual cognitive behaviour therapy in the treatment of panic disorder and agoraphobia in primary care. Clinical Psychology and Psychotherapy, $11,73-82$.

Starcevic, V., Uhlenhuth, E.H., Kellner, R., y Pathak, D. (1992). Patterns of comorbidity in panic disorder and agoraphobia. Psychiatric Research, 42, 171-183.

Spielberger, C.D, Gorsuch, R.L., y Lushene, R.E. (1994). STAI-Cuestionario de ansiedad estado-rasgo. Madrid: TEA.

Stuart, G.L., Treat, T.A., y Wade, W.A. (2000). Effectiveness of an empirically based treatment for panic disorder delivered in a service clinic setting: 1-year follow- up. Journal of Consulting and Clinical Psychology, 68, 506-512.

Telch, M.J., Lucas, J.A., Schmidt, N.B., Hanna, H.H., LaNae Jaumez, T., y Lucas, R.A. (1993). Group cognitive-behavioral treatment of panic disorder. Behavior Research and Therapy, 31, 279-287.

Wade, W.A., Treat, T.A., y Stuart, G.L. (1998). Transporting an empirically supported treatment for panic disorder to a service clinic setting: A benchmarking strategy. Journal of Consulting and Clinical Psychology, 66, 231239.

Weissman, M.M. (1991). Panic disorder: Impact on quality of life. Journal Clinical Psychiatry, 52, 6-8.

Weissman, M.M., Bland, R.C., Canino, G.C., Faravelli, C., y otros (1997). The cross-national epidemiology of panic disorder. Archives of General Psychiatry, 54, 305-309.

Weissman, M.M., Klerman, G.L., Markowitz , J.S., y Oullette, R. (1989). Suicidal ideation an attempts in panic disorder and attacks. New England Journal of Medicine, 321, 1209-1214.

Wolfe, B.E., y Márser, J.D. (1994). Treatment of panic disorder: a consensus development conference. Washington DC: American Psychiatric Press.

Yonkers, K.A., Zlotnick, C., Allsworth, J., Warshaw, M., Shea, T., y Keller, M.B. (1998). Is the course of panic disorder the same in women and men? American Journal Psychiatry, 155, 596-602. 